\title{
Turín, Ciudad Educadora
}

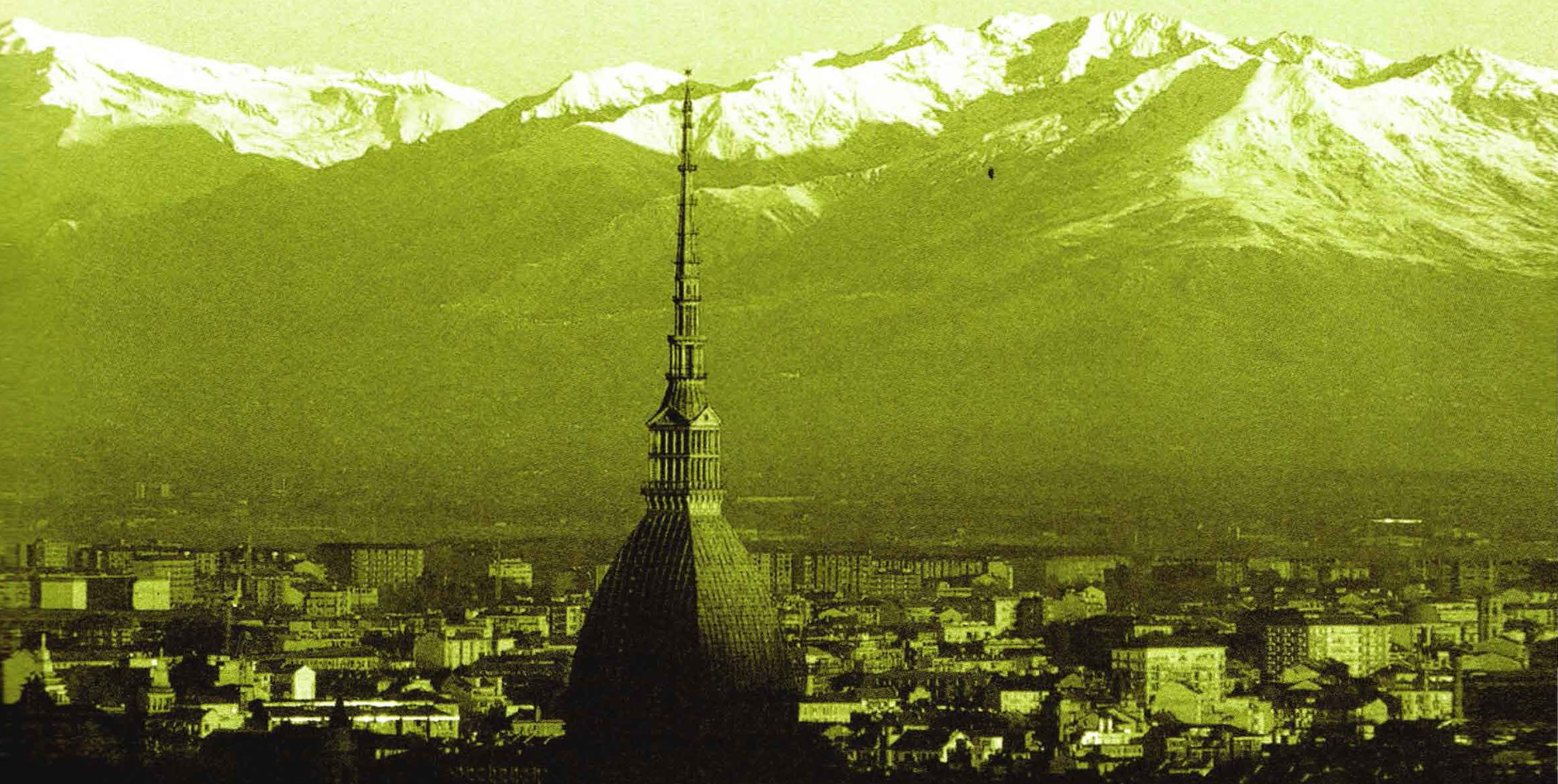




\section{Fiorenzo Alfieri}

Graduado en psicología, realizó estudios laureados en pedagogía. Ha sido dirigente escolar. Catedrático en psicología del desarrollo en la Universidad de Turín. Asesor de la Alcaldía de Turín en deporte, juventud, tiempo libre y turismo. Entre 1995 y 1997, consejero de Educación de TorinoTurín, y de 1997 a 2000, consejero de la Secretaría de Comercio de esa misma ciudad. Obtuvo la condecoración "Honor al Mérito" por parte de la República italiana.

\section{Resumen}

Este texto traza un recorrido por la evolución de Turín, Italia, como ciudad y como Ciudad Educadora desde el Renacimiento hasta la época actual. Muestra su desarrollo a lo largo de la historia y cómo su dinámica industrial, tecnológica, científica, urbanística, social, comercial y educativa ha propiciado modelos educativos que vinculan a la escuela con la ciudad y viceversa. Asimismo, el autor muestra su experiencia en términos de agente activo y presenta distintos vínculos a los que se puede acceder a través de la página Web que él administra.

\section{Palabras clave}

Turín, Freinet, información, alcaldía, servicios, innovación, transformación, Ciudad Educadora.

\section{Abstract}

This article walks through the evolution of Turin, Italy as a city and as an Educating City since Renaissance and up to the present times. It shows its historical development and how its industrial, technological, scientific, urbanistic, social, commercial, and educational dynamics have provided educational models that tie the scool with the city and viceversa. Concurrently, the author evidences his experience as an active agent and shows diverse links that can be visited through the Website he manages.

\section{Key words}

Turin, Freinet, information, Mayor's office, Services, innovation, transformation, Educating City. 

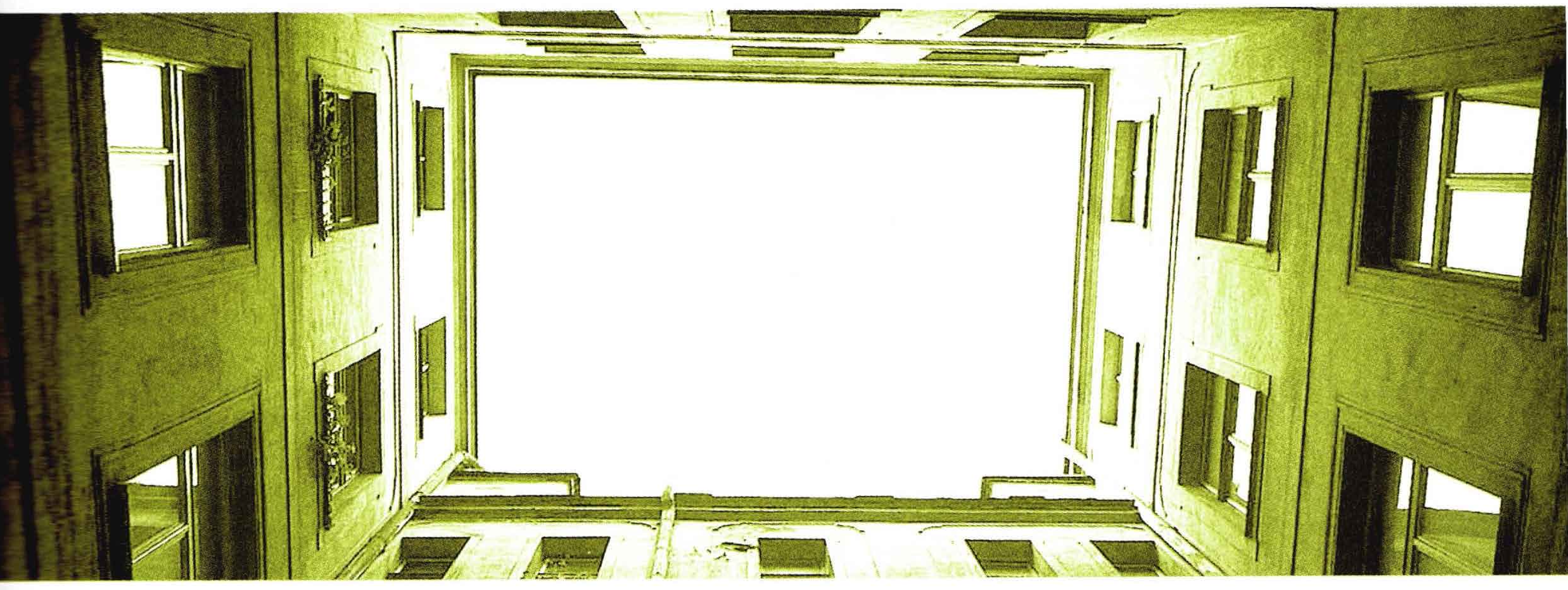

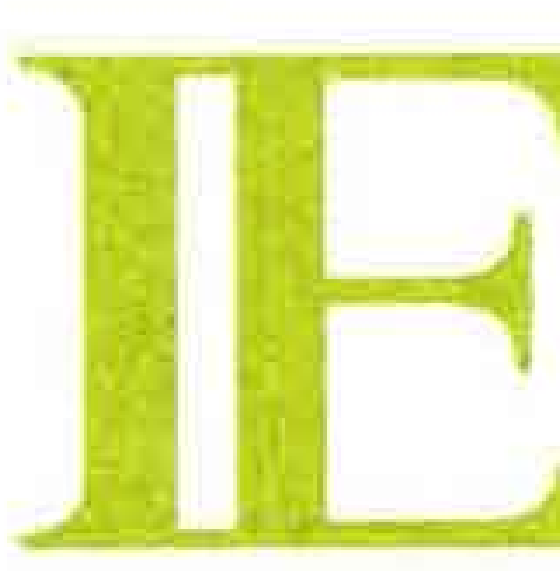

n Italia, Turín es por antonomasia la ciudad-laboratorio. Casi todo nació allí y se extendió luego a nivel nacional. Desde mediados de 1500 hasta 1861 fue la capital del único Estado autónomo italiano, pues los otros territorios estaban ocupados por potencias extranjeras. En Turín se ha formado la cultura de Estado que, después de 1861, se extendió por todo el país. El Estado autónomo, con Turín como capital, desarrolló una política militar que contribuyó a la unificación de Italia, y en 1861, fue su primera capital. Cuando fue desplazada por razones geográficas, antes a Florencia y después a Roma, tuvo que concebir un nuevo porvenir, pues mientras fue capital del pequeño Estado, debió desarrollar técnicas militares eficaces para la defensa de otros Estados europeos -entre ellos la cercana Francia-; además, se asistía a un fuerte desarrollo de las ciencias y sobre todo de las tecnologías. Turín fue la primera ciudad italiana que hizo importantes esfuerzos para el desarrollo de la industria textil y de la metalmecánica, convirtiéndose así en la capital indus- trial del pais, y antes que en otras ciudades el automóvil, la gran moda, el cinema, la radio y más tarde la televisión encontraron lugar. Por ello, en la segunda posguerra, a la riqueza de innovaciones sucedió una vocación casi exclusiva para la industria manufacturera fuertemente relacionada con la del automóvil.

Entre las nuevas propuestas nacidas en Turín, ocupa un primer lugar la relación entre escuela y ciudad. Esta idea adquirió forma en 1975 -precisamente hace treinta años-, pero no fue algo inesperado: quince años atrás en muchas escuelas primarias de la ciudad se había desarrollado una didáctica innovadora inspirada en las técnicas de Célestin Freinet, promovidas en Italia por el Movimento di Cooperazione Educativa, MCE. Dichas técnicas se basaban en el pensamiento del filósofo americano John Dewey que, sobre todo en su obra Escuela y sociedad, planteaba un criterio fundamental para distinguir la calidad de la escuela: todas las actividades desarrolladas en ella deben repercutir en el mundo exterior; es decir, en la sociedad. Según afirmaba Dewey, la escuela tiene que pre- 
parar para la vida y el único instrumento didáctico que sirve es la vida misma. Del pensamiento de Dewey se desarrolló el movimiento de la Escuela Activa, en el que las técnicas de Freinet adquirieron una importancia especial debido a su originalidad y aplicabilidad concretas. En Italia y hasta la mitad de los años sesenta, el Movimento di Cooperazione Educativa se había enraizado sobre todo en centros pequeños, caracterizados por grupos de clase reducidos y problemas sociales moderados. Sucedió un cambio sensible cuando al MCE se acercaron profesores -como yo y muchos otros- que trabajaban en barrios de las afueras de las grandes ciudades del norte. Se registraba un crecimiento tumultuoso de la población debido al gigantesco fenómeno de emigración del sur, que involucraba poblaciones enteras que se desplazaban para trabajar en las grandes fábricas metalmecánicas. En poco más de una década la población se duplicó - pasando de $600.000 \mathrm{mil}$, en la posguerra, a 1.250 .000 en $1975-$, a la que fue añadiéndose por lo menos otros quinientos mil de la periferia. Aplicamos las técnicas Freinet -entre la segunda mitad de los años sesenta y la primera mitad de los setentaen clases con muchos alumnos, exclusivamente recién inmigrados, y de ellas derivó una práctica social que se propagó automáticamente de la escue- la a las familias y a los distritos. Las primeras experiencias de los comités de barrio se desarrollaron en las zonas de trabajo de los profesores del MCE. Entre las técnicas Freinet tradicionales -texto libre, tipografía en clase, diario de la escuela, carteo entre escuelas lejanas, actividades artísticas, estudio medioambientaleste último tuvo un éxito especial. Antes de todo era necesario entender la nueva realidad en la que los chicos vivían, y crear una nueva ciudadanía, una nueva conciencia civil.

En 1975 tuvieron lugar las elecciones administrativas. Algunos de nosotros, que nos habíamos consagrado a practicar ese método de enseñanza, resultamos elegidos y designados para llevar a cabo las políticas escolásticas y juveniles. Para nosotros fue natural pensar que si se quería favorecer el crecimiento de ciudadanos democráticos y responsables, era necesaria la intervención de la ciudad; es decir, la administración comunal cercana al Instituto

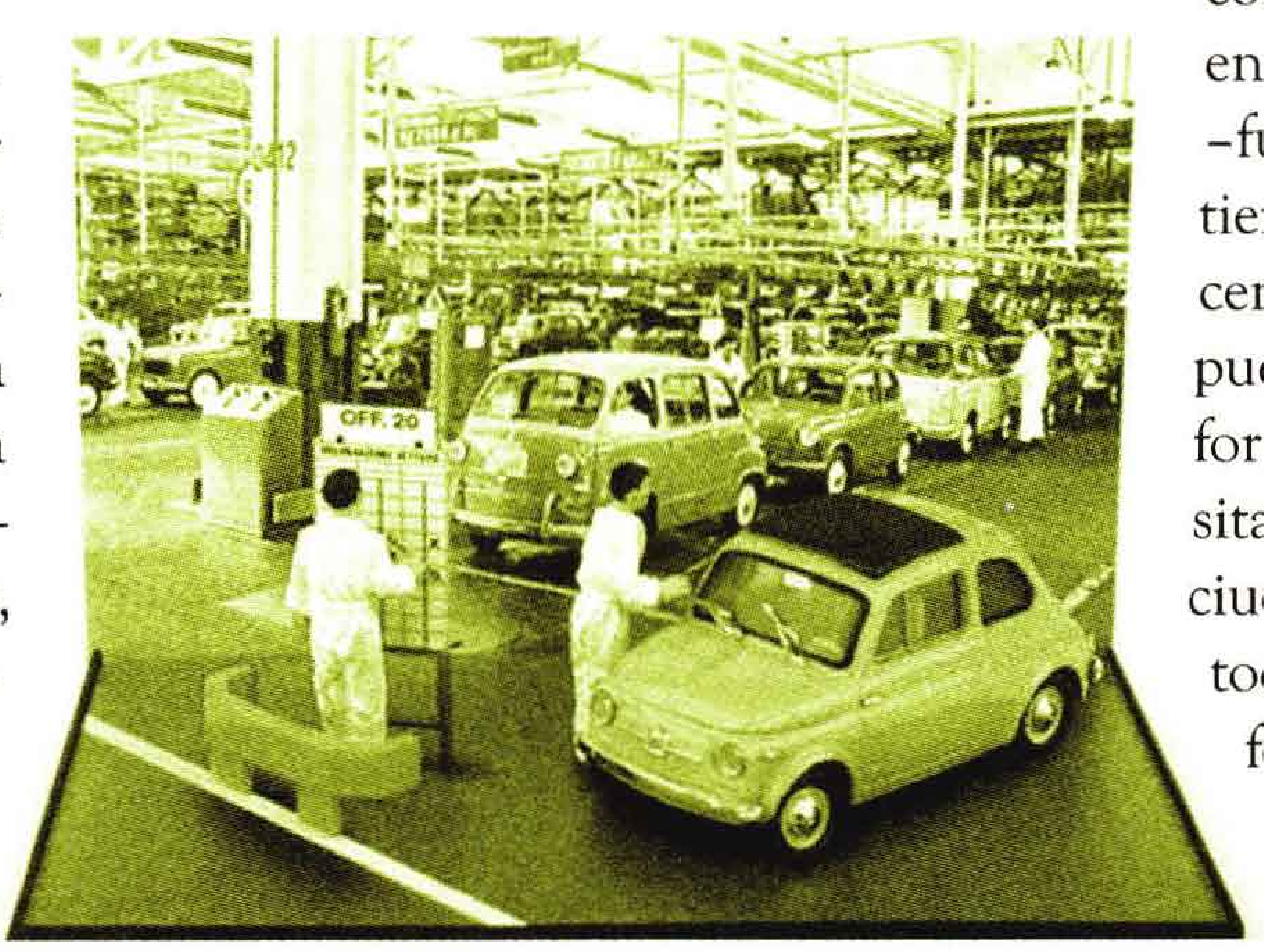
en el que todos los muchachos -futuros adultos- pasaban un tiempo de su niñez y adolescencia. Pero la escuela sola no puede desempeñar la función formativa que la sociedad necesita; es imprescindible que la ciudad ponga, a su disposición todos, los recursos formativos, formales e informales.

Desde el perfil pedagógico, esta visión desta- 


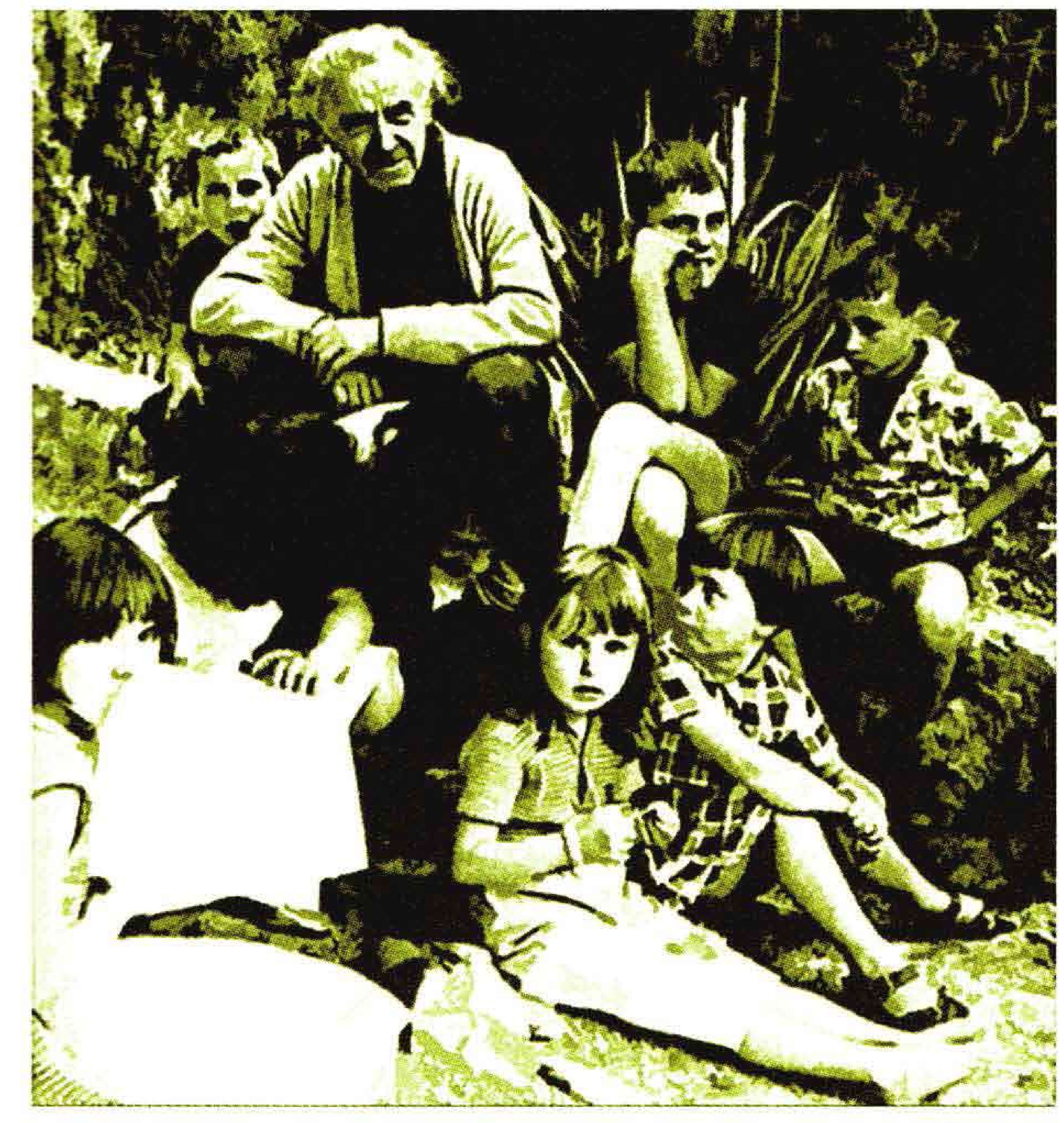

Freinet

caba un elemento nuevo muy importante, también con respecto a la didáctica más innovadora, incluida la inspirada en Freinet. En realidad, era importante que los niños experimentasen un contacto directo con la naturaleza; entonces preparamos un huerto, un jardín, un acuario y un pequeño zoológico. Era importante que los niños vivieran el teatro, el cinema o la danza y de acuerdo con ello creamos en la escuela un pequeño teatro.

En 1975 desarrollamos una idea revolucionaria: la ciudad podía ofrecer a la escuela espacios y oportunidades normales, y no en miniatura, con el fin de enriquecer las potencialidades formativas de las escuelas y al mismo tiempo hacer comprender, amar y defender, desde la perspectiva de los adolescentes, su nueva ciudad. El alcalde de entonces, Diego Novelli, y los concejales nos dirigimos antes que a las escuelas y a los profesores, a los distintos grupos extra-escolásticos de la ciudad con estas palabras: "El correcto funcionamiento de nuestro sistema educativo representa un interés común; la escuela no puede sola; cada uno de ustedes debe prepararse para dar su aporte contribuyendo con situaciones y personas que puedan revelarse como útiles e interesantes". Muchos grupos del sistema-ciudad reaccionaron positivamente, pues se transmitió el principio de que la educación de las nuevas generaciones interesaba, en el largo plazo, no sólo y no tanto a la escuela, sino también y en particular a esos grupos. Los teatros tenían interés por preparar públicos nuevos; las bibliotecas porque los ciudadanos se apasionaran por la lectura desde niños; las industrias porque el ciclo productivo se conociera y valorara; las asociaciones deportivas porque la gente se acostumbrara a amar el ejercicio físico dirigido; las organizaciones naturalistas porque se desarrollara el conocimiento y el respeto por las plantas y animales; los bomberos porque los ciudadanos hicieran una prevención adecưada; la asociación de panaderos porque se valorara el buen pan; y hasta la Guardia Civil y la Policía porque que fueran concientes y respetuosos de su trabajo.

Como decía, no fue difícil reunir las disponibilidades de los tantos grupos del sistema-ciudad: cada uno eligió los contenidos, el tiempo y los métodos para desarrollar las actividades que podía ofrecer a las escuelas. El Ayuntamiento recogió las propuestas, clasificándolas por argumento y edad y ofreciéndolas, al comienzo de cada año, a las escuelas para 
sus distintos grados. La relación con las escuelas no presentó la misma facilidad registrada en el caso de las organizaciones de ciudadanos. Las escuelas que experimentaban nuevas técnicas didácticas estaban acostumbradas a verse

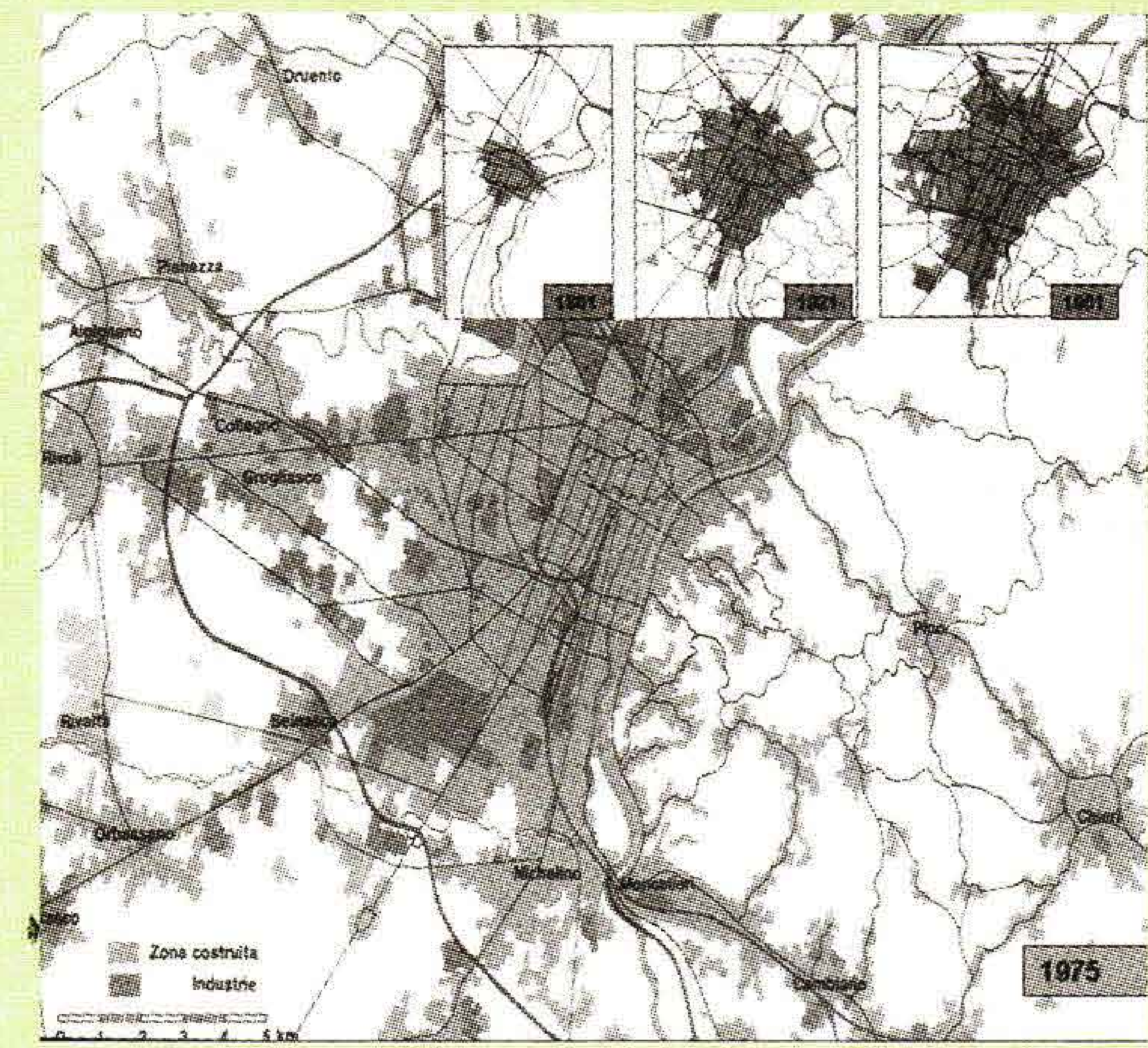
como microcosmos dotados de todos los recursos necesarios para una educación de tipo activo; las otras - menos innovadoras- temían a la idea de salir de su estructura y enfrentarse con la complejidad y los "peligros" de la ciudad. Pero la oposición política nos culpó de inmiscuirnos en la escuela pública -un sector que excedía la competencia directa de la ciudad-, con el fin del adoctrinamiento ideológico de niños y familias.

Precisamente los grupos extra-escolásticos convencieron, poco a poco, a los profesores de la utilidad de sus propuestas, que en nada se aproximaban a ideologías o "peligros", sino que solamente aspiraban a hacer conocer de primera mano muchos perfiles de la ciudad a las personas que estaban preparándose para ser los ciudadanos adultos del mañana.

Siempre insistimos en que las situaciones de contacto entre escuela y ciudad permitieran a los niños y adolescentes conocer directamente a los sujetos que trabajaban en la ciudad, de modo que pudieran identificar de la forma más clara posible las características peculiares de las distintas actividades y formas de "inteligencia". El gusto por los museos, campos deportivos, teatros, laboratorios de investigación, viveros, servicios técnicos, estaciones de bomberos, guardias municipales, fábricas, mercados, etcétera, exigía oportunidades no sólo de "visita escolástica”, sino también de contacto directo con educadores naturales -otros profesores-, capaces de transmitir el interés por el trabajo, la investigación, la creación y las responsabilidades. El profesor no debía sustituir a todos estos educadores naturales y "entusiastas", sino que debía convertirse en un eficaz intermediario cultural entre los estudiantes y los distintos grupos del sistema-ciudad. Así, la experiencia ha demostrado de manera sorprendente la veracidad de la idea de Dewey, según la cual el mejor instrumento didáctico de preparación a la vida es la vida misma.

Desde la prospectiva del sistema-ciudad, los años empleados en el fortalecimiento de contactos directos entre las escuelas, los niños y la ciudad han hecho que las instituciones, asociaciones, empresas y los distintos grupos desarrollaran la costumbre y el interés por la comunicación social en el sentido más amplio del término. Estamos hablando de un fenómeno muy sutil que necesitaría de una investigación atenta.

A los que llegan a nuestra ciudad y empiezan a conocerla, les asombra la atención puesta en cada esquina para la información y la participación de 
los ciudadanos, la cual está encaminada a la "satisfacción del cliente". En toda obra pública existen paneles con la historia del edificio, las características del proyecto, el tiempo y los gastos de realización, el resultado final, las disculpas por las molestias causadas. Toda intervención en la viabilidad o en otro aspecto de la vida cotidiana de los ciudadanos, exige una preparación que se compone de encuentros públicos y distribución profusa de material informativo. Y así por el estilo. Esta característica de la calidad de vida en el Turín de hoy se basa precisamente en los treinta años pasados, los cuales fueron dedicados a investigar lo que puede ser importante, desde el perfil educativo, en el tejido profundo de la ciudad. Y con eso no quiero decir que los ciudadanos estén satisfechos del nivel de comunicación existente: precisamente la experiencia que les he contado ha creado expectativa en los ciudadanos para que reaccionen con prontitud y señalen a las administraciones públicas y a los medios de comunicación lo que no funciona o lo que no ha sido suficientemente difundido.

- De la gran cantidad de trabajo realizado en los primeros años surgió una idea aun más ambiciosa: transformar la ciudad - puesta a disposición de la escuela- en una verdadera Ciudad Educadora. Se trata de dos hipótesis más bien distintas. En el primer caso es necesario entender la escuela como único punto de referencia para que los niños y los jóvenes desarrollen un contacto directo con los variados grupos del contexto urbano. De hecho, las escuelas

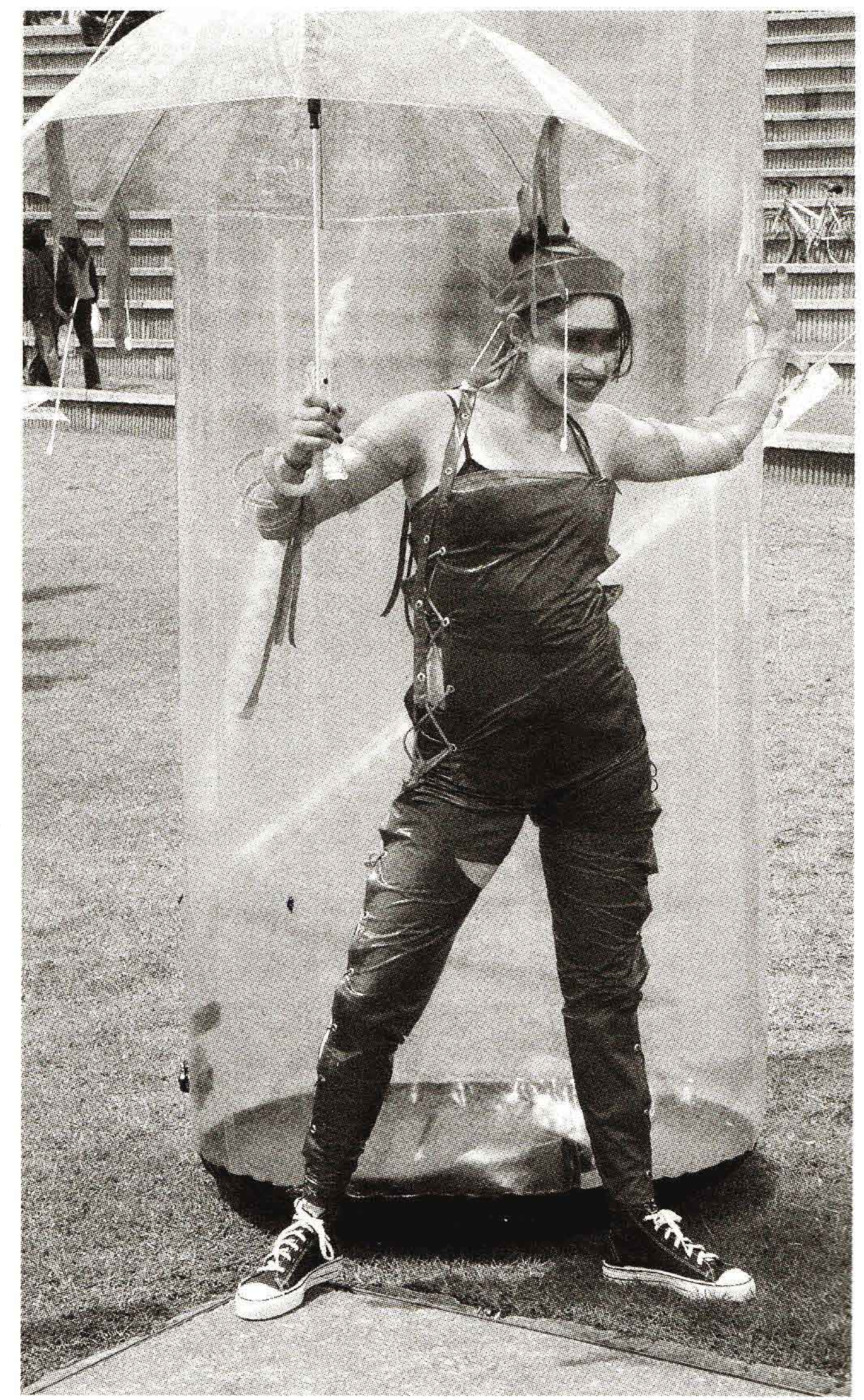


la escuela se difundian con relativa dificultad, en Cataluña y por toda España la difusión fue extraordinariamente rápida. Por una parte, empezó el proceso que dio lugar a la gran reforma escolar en España -desde la guardería infantil a la universidad-, y de otra parte, se desarrolló la idea de Ciudad Educadora. Y precisamente en Barcelona, en noviembre de 1990, tuvo lugar el primer congreso mundial sobre este tema que concluyó con la adopción de la Carta de las Ciudades Educadoras, documento que sentó las bases para la fundación de la Asociación Internacional de las Ciudades Educadoras, AICE. Turín fue ciudad socia fundadora y sigue siendo miembro del Comité Ejecutivo de la AICE. Aunque Turín abrió las puertas al proceso mental, cultural y organizativo que contribuyó a difundir la idea de "Ciudad Educadora", la fuerza necesaria para la conversión en movimiento internacional vino de una ciudad como Barcelona y de un país como España, en donde las políticas educativas asumieron un papel principal único con respecto a lo que se ha podido realizar en Italia.

' Es típico de Italia elaborar, en todos los sectores, experiencias significativas, inteligentes e innovadoras, pero al mismo tiempo encontrar dificultades para transformarlas en estructuras básicas de la organización social. El efecto producido son acciones experimentales muy elevadas, por un lado, y estándares generalizados de calidad muy inferior, por otro.

Turín sufrió un período de hastío correspondiente al cambio de la mayoría política en el gobier- no de la ciudad -entre 1985 y $1993-$, que fue caracterizado por la administración de alcaldes distintos. Después de la reforma electoral de 1993, en Italia los ciudadanos comenzaron a elegir por voto directo al alcalde y se abrió una época de mayor estabilidad política. En mi caso, después de trabajar como concejal para la juventud, de 1976 a 1985, fui otra vez concejal -para el sistema educativo esta vez-, en 1995, y sigo siendo miembro del gobierno de la ciudad como concejal para la cultura.

En lo que se refiere a la primera iniciativa de que he hablado, en cuyo ámbito Turín puso a disposición de las escuelas todas las posibilidades ofrecidas por el sistema-ciudad, después de treinta años la experiencia sigue adelantándose y se desarrolla más y más, año tras año. Sigo insistiendo en que el proyecto se llama hoy Crescere in città, con el subtítulo de Attività educative rivolte a bambini, ragazzi e adulti. Muchos años después de su difusión mediante un gran catálogo de oportunidades, que se distribuía en las escuelas en los primeros días de septiembre de cada año escolar, actualmente se ha establecido un sitio en la Internet con la dirección: www.comune.torino. it/servizi-educativi/crescere-in-città. Lo consultamos rápidamente en la edición del año escolar 2004. 2005, ya que la versión relativa al siguiente año escolar no estaba todavía disponible al momento de preparar esta intervención. En el sitio Web están presentes 800 actividades educativas, a las que se les añade 21 reseñas teatrales para un total de 207 espectáculos. 


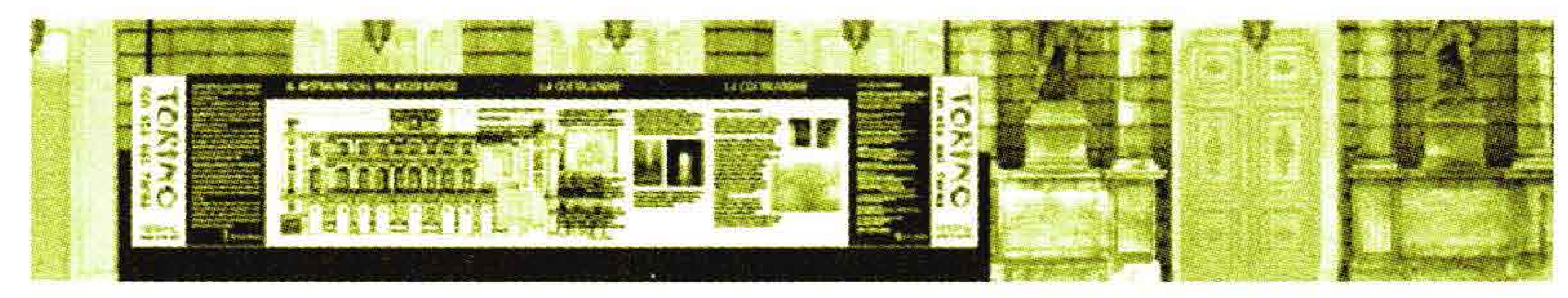

Para obtener información sobre los servicios que la ciudad de Turín pone a disposición del sistema educativo hoy, una vez en la página de Internet "clicamos" en Informazioni generali y después en la Carta dei servizi. En primer plano encontramos las estructuras básicas que la ciudad administra directamente:

- 49 guarderías infantiles y 30 micro-guarderías. El Estado no provee estos servicios; los cuales no son municipales sino privados.

- 85 escuelas maternas -son más o menos la mitad de las existentes-; las otras son públicas o concertadas con el Ayuntamiento. La ley no obliga a los municipios a administrar las escuelas maternas. La ciudad de Turín actúa de esta manera por razones políticas; en efecto, la ciudad no quiere olvidar la tradición gloriosa de nuestras escuelas que siempre han sido consideradas como las mejores de Italia.

- 4 centros de educación especial, equipados especialmente para minusválidos graves.

- 6 centros lúdicos, como integración del servicio de las guarderías infantiles.

- 10 centros para familias.

- 3 puntos para familias.

La siguiente lista relaciona las estructuras y los proyectos que integran la oferta:
- 8 institutos de cultura. Cada uno en un edificio adecuado dividido en distintas secciones de trabajo, a disposición de las escuelas en horario escolar y de las familias fuera del horario escolar.

- 9 proyectos educativos.

- 6 ludotecas y centros lúdicos.

- 8 salas de juego en el hospital.

- 1 granja urbana.

- 1 centro multimedial de documentación pedagógica.

- El centro de estudio teatral juvenil, G. R. Morteo.

- Asesoramiento educativo domiciliario, CED.

- La oficina Un anno per crescere insieme.

- Un centro mundial para extranjeros y nómadas.

- La oficina para viajes e intercambios escolares internacionales.

- El laboratorio Ciudad sostenible para las niñas y niños.

- Dos laboratorios didácticos sobre el medio ambiente: Loano y Pracatinat.

- Casa marina La vela.

- Un centro para promoción de servicios educativos y organización.

Para formarse una idea aproximada, es interesante clicar en Proposte di percorsi disciplinari. Esa sección incluye los resultados -a partir de las prime- 
ras experiencias descritas-, que establecían como objetivo poner a disposición de las escuelas todas las oportunidades educativas presentes de manera más o menos implícita en el sistema-ciudad. Se observa que las oportunidades están divididas en:

- Educación medioambiental.

- Educación alimentaria.

- Educación científica.

- Educación ciudadana.

- Educación en salud.

Hay dos opciones en la página Web: la de la izquierda ofrece opciones dirigidas a adultos: profesores, padres y educadores; la otra muestra las actividades con niños, adolescentes y adultos. Clicando en esta última se encuentra la cantidad de propuestas anuales a disposición de los jóvenes.

En la columna de la izquierda del sitio destacan las áreas equipadas donde se realizan las experiencias y los proyectos; entre todos, algunos son puestos de manera particular. Cerca de 300 personas trabajan en el ámbito de las experiencias y de los proyectos.

Y ahora unos ejemplos. Clicamos en Educazione all'Identità e alle culture. Se trata de uno de los "Institutos de Cultura" cuya presentación es la siguiente:

"El impulso al diálogo con niños y adolescentes para atrapar pensamientos, expectativas, preguntas sobre sí mismos, los otros, la ciudad, el pasado, el futuro.
- La implementación de oportunidades educativas para aprender a ser, elegir, evaluar, decidir, conocerse, adaptarse, cambiar.

- Desarrollar la convicción de que los pensamientos, las maneras de ser, el encuentro con los otros determinan la identidad de cada uno.

- La educación para la investigación con el objeto de adquirir una personalidad abierta e interactiva y favorecer el desarrollo original de las personas y de las culturas que interaccionan.

- Las reflexiones sobre la creación de una sociedad intercultural, como proyecto concreto y realizable desde una perspectiva de discusión y superación de los etnocentrismos".

Estos y otros objetivos mueven las acciones que el Centro propone a los niños y adolescentes a través de las secciones de investigación: organización social, trabajo, ritos y rituales, literatura y música. Así, la cultura que se explora es la de ayer y la de hoy -la cultura cercana-, la cultura del mundo. Se quiere ofrecer a niños y adolescentes visiones extendidas de cultura, multiettnicidad en las relaciones, para que se transformen en "ciudadanos del mundo".

La página continúa con indicaciones sobre horarios de clase -de 8:30 a 13:00 de lunes a viernes-; la dirección del Centro -hay también un mapa, nú-

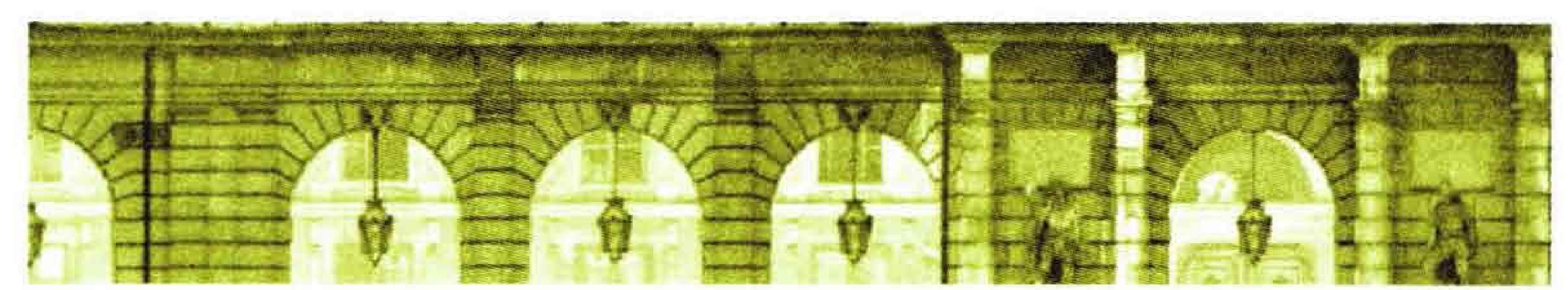




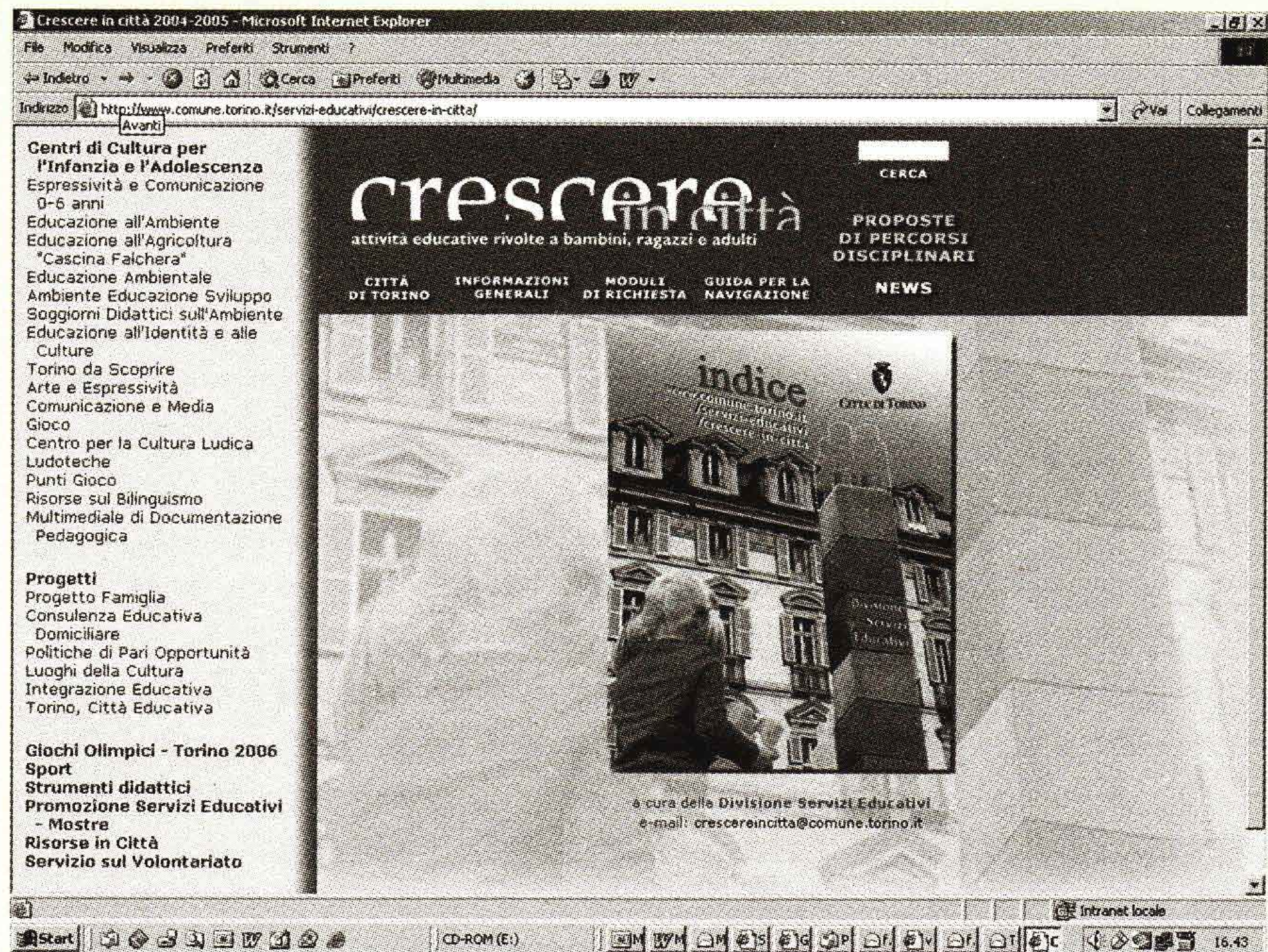

meros de teléfono y fax, dirección de correo electrónico-; de los horarios de asesoramiento -miércoles y viernes por la tarde de $14: 30$ a 17:00 previa cita telefónica-, y de los horarios al público: lunes, miércoles y jueves por la tarde de 14:30 a 18:00.

El Centro ofrece dos exposiciones interactivas para distintas edades y se pueden experimentar itinerarios diversos en lo referido a organización social, trabajo, ritos y rituales, literatura y música. Cada itinerario, dividido en varias secciones, está descrito de manera que los profesores puedan conocer su contenido antes de empezar. Por otra parte, muchas actividades incluyen encuentros preliminares con los profesores.

Descubrimos otro Centro más; el llamado "Torino da Scoprire”. Esta es su presentación:
"Una mirada alrededor, la búsqueda de la memoria de la comunidad en que se vive, el conocimiento de rasgos sugestivos, la búsqueda de ráíces comunes, todo esto significa operar en el mundo para que se construya una identidad propia capaz de dialogar y estar a la altura de los otros".

En lo atinente a este tema, Turín tiene una rica tradición expresada en los laboratorios sobre el conocimiento de la ciudad y del Piamonte, y en los intercambios escolares, hasta concretizarse recientemente en la creación de un instituto de cultura equipado para conocer y comprender la historia y las repercusiones de la actividad del hombre en la región.

El rasgo principal del "Centro Torino da Scoprire" es el estudio de la memoria colectiva, teniendo conciencia sobre el hecho de que la creación del sujeto-persona comprende la autobiografía de la región, en la que las historias particulares se funden con la historia de la ciudad y el elemento local se integra con el global. No hay contradicción entre apertura a la novedad, a la mundialidad, al amor y al orgullo por los orígenes, pero este objetivo se consigue viviendo experiencias educativas capaces de dar sentido a la palabra "comunidad".

El conocimiento del pasado se mezcla también con el conocimiento de los hechos posibles de un futuro cercano, de las raices de nuestra historia para 
llegar a comprender la ciudad del tercer milenio: sus cambios, su aptitud para manejar situaciones medioambientales complejas. Se trata de un camino de desarrollo civil y cultural, en el que los niños están invitados a participar en tiempo real con respecto a los cambios en curso.

Algunas secciones son Torino, turismo scolastico, Piamonte -la región cuya capital es Turín-, y La scuola adotta un monumento, entre otras.

Los objetivos son:

- Valorar, promover y tutelar el patrimonio histórico-artístico de nuestra ciudad.

- Estimular a los alumnos a la acción, a la participación y a la responsabilidad de su adopción.

- Conocer y hacer conocer los monumentos adoptados por las escuelas de Turín presentes en el área urbana.

\section{El proyecto incluye tres fases}

\section{Primera}

- Prevé que los estudiantes estudien, con la ayuda de los profesores, los distintos rasgos y características del monumento -históricoiconográficos, urbanístico-medioambientales, sociales-, a la luz de las dimensiones transculturales y transnacionales. c i u d a d e d u c a d o r a

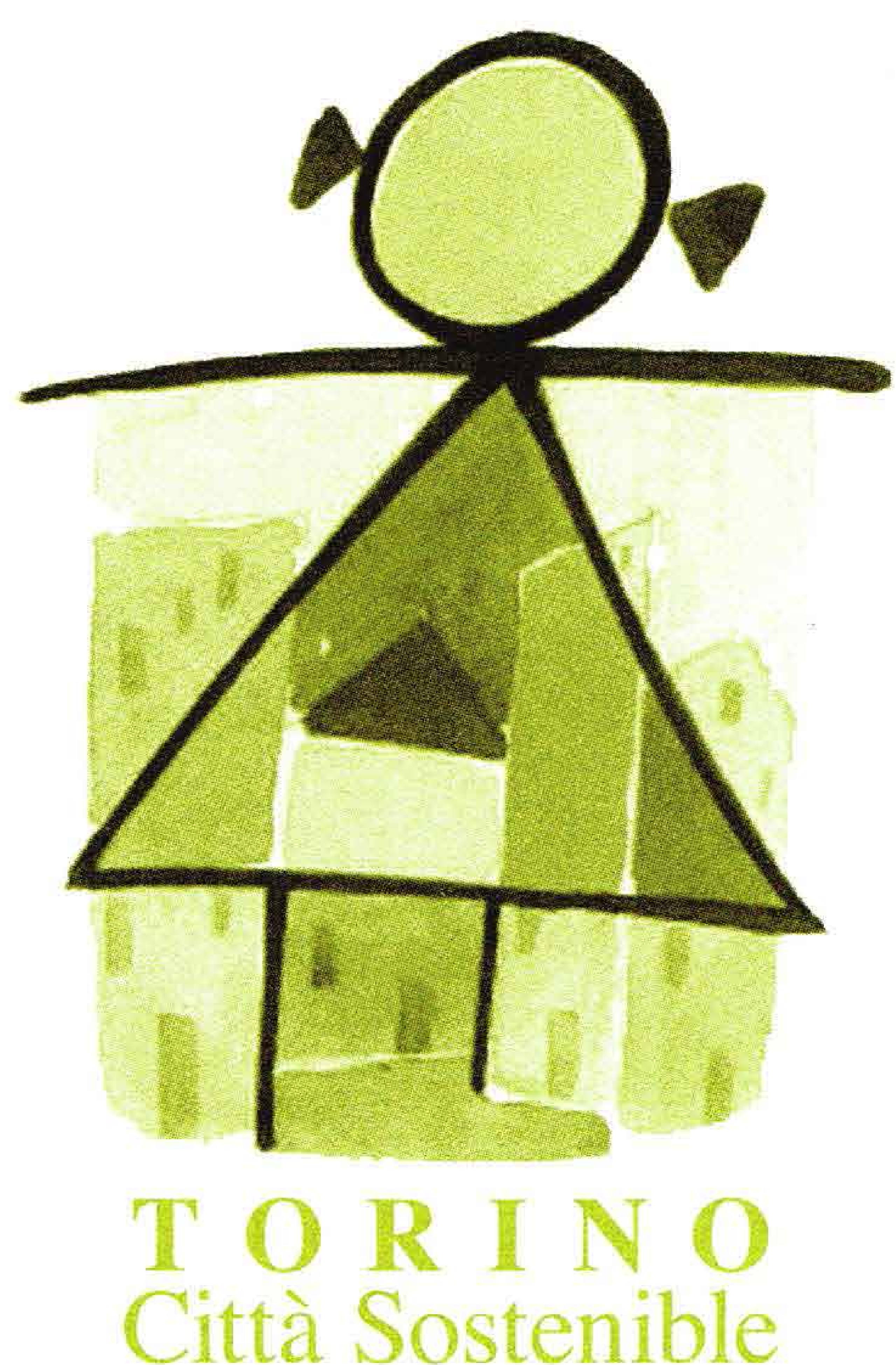

- Se puede adoptar un monumento "tradicional”: iglesias, museos, edificios históricos, fuentes, estatuas, etcétera; o un área de interés especial: plazas y calles, aglomeraciones urbanas homogéneas que juegan un papel histórico-afectivo importante para la comunidad.

\section{Segunda}

- Una vez terminada la fase de investigación se elaborará un proyecto de valoración del monumento elegido con la producción de material de divulgación, y actividades de publicidad y promoción que 
permitan, además, un mayor gusto por el bien cultural.

- En el ámbito del proyecto es posible proponer hipótesis de recalificación de las áreas de los monumentos adoptados, con un trabajo que permita la reevaluación del bien cultural a través de un nuevo proyecto del tejido urbano para reafirmar la identidad del sitio monumental.

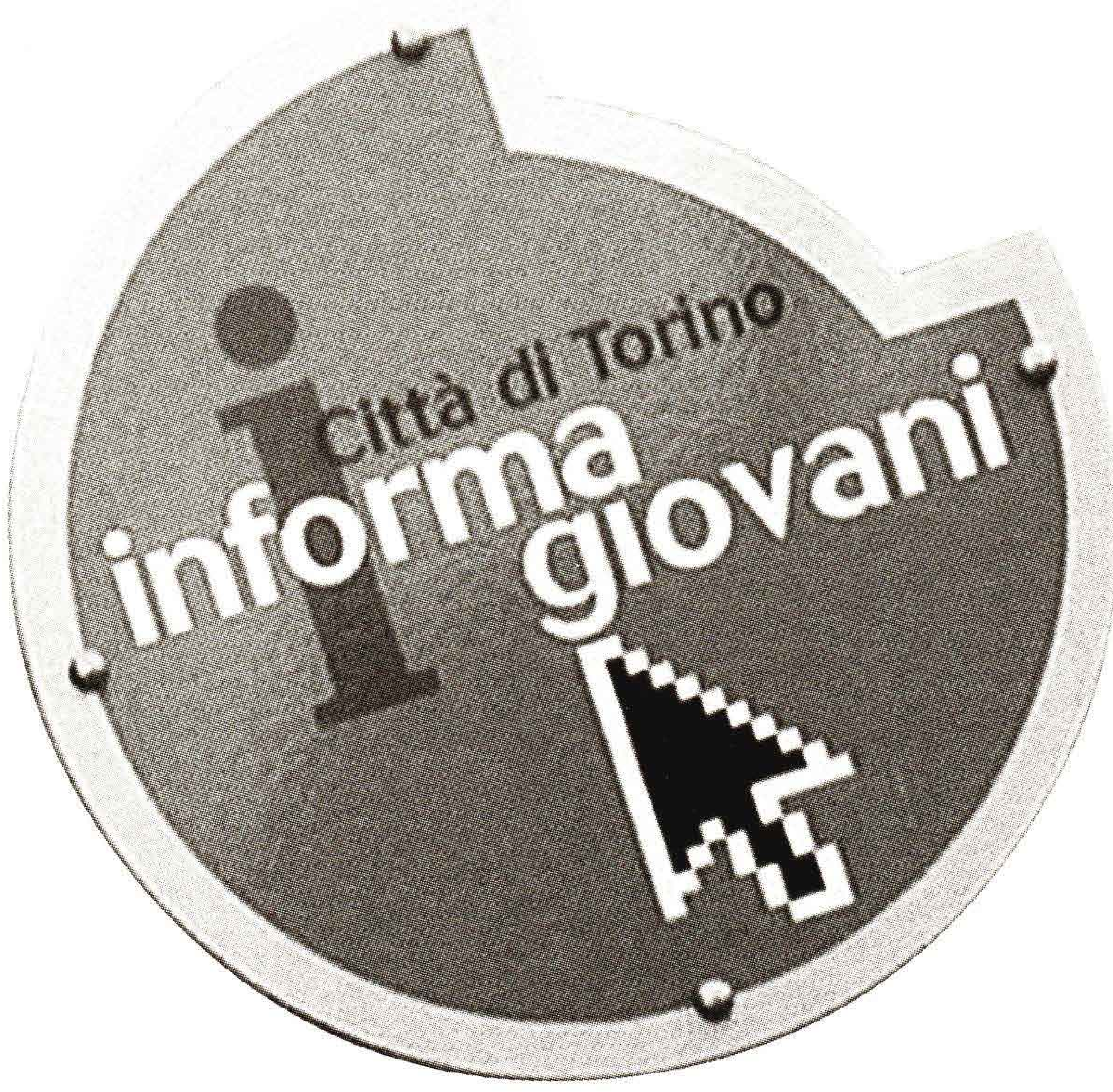

\section{Tercera}

- Prevé una serie de interacciones entre las escuelas que participan en el proyecto d nivel nacional, con la realización de eventuales hermanamientos, de intercambios de los materiales realizados, organización de visitas a los monumentos adoptados y creación de itinerarios culturales educativos.

- Durante todo el período de adopción, la escuela se beneficiará con el gusto privilegiado por el monumento y se sentirá responsable de vigilarlo simbólicamente; igualmente servirá de guía en eventos públicos de interés especial o en días establecidos para las visitas de otros institutos o grupos organizados.

\section{Propuesta Torino porte aperte}

- Cada año las escuelas que participan en el proyecto toman parte en Torino porte aperte, que incluye la apertura al público de los monumentos adoptados.

- Los niños y los adolescentes actúan como guías entusiastas para los ciudadanos, que descubren los monumentos adoptados y el deleite de pasear a la búsqueda de las bellezas de nuestra ciudad.

Profundizaremos en uno de los proyectos, el "Progetto famiglia", cuya descripción es la siguiente: 


\section{cresceringtà \\ attivita educative rivolte a bambini, ragazzi e adulti}

El "Progetto famiglia" dirige sus propuestas a los padres de las niñas y de los niños del grupo de 0 a 6 años, y ofrece oportunidades formativas y de divulgación a una pluralidad de sujetos diferentes en sus deseos y exigencias. Este proyecto se integra a la red de servicios como nodo para crear ocasiones de intercambio y discusión de experiencias entre familias, y propone actividades formativas destinadas a educadores y profesores de guarderías infantiles y escuelas maternas, así como a los participantes de los itinerarios educativos.

Asimismo, organiza encuentros y seminarios de profundización teórica en asuntos psicopedagógicos, jurídicos, sanitarios, culturales y sociales como:

- Organización y coordinación de las actividades.

- Sitio Web con actualización periódica de las propuestas.

- Primer contacto telefónico e indicación a las oficinas competentes.

- Reservación de las propuestas.

- Información.

- Banco de datos.
- Biblioteca para padres.

\section{Puntos de información para las familias}

Se sitúan en los servicios educativos municipales -guarderías infantiles y escuelas maternas-, uno por cada distrito de la ciudad. La propuesta incluye encuentros, cursos, actividades de grupo no sólo para ofrecer ayuda e instrumentos de trabajo a las familias, sino para crear intercambios, encuentros y discusiones teniendo en cuenta su papel único en la relación con los hijos:

- Informaciones sobre servicios y actividades para familias propuestas en la ciudad.

- Asesoramiento educativo y didáctico para los padres.

- Formación mediante encuentros temáticos con expertos en asuntos educativos, sociales, sanitarios y jurídicos.

- Discusión en grupos guiados.

- Encuentros con actividades conjuntas de laboratorio para niños y padres, en colaboración con los institutos de cultura. 


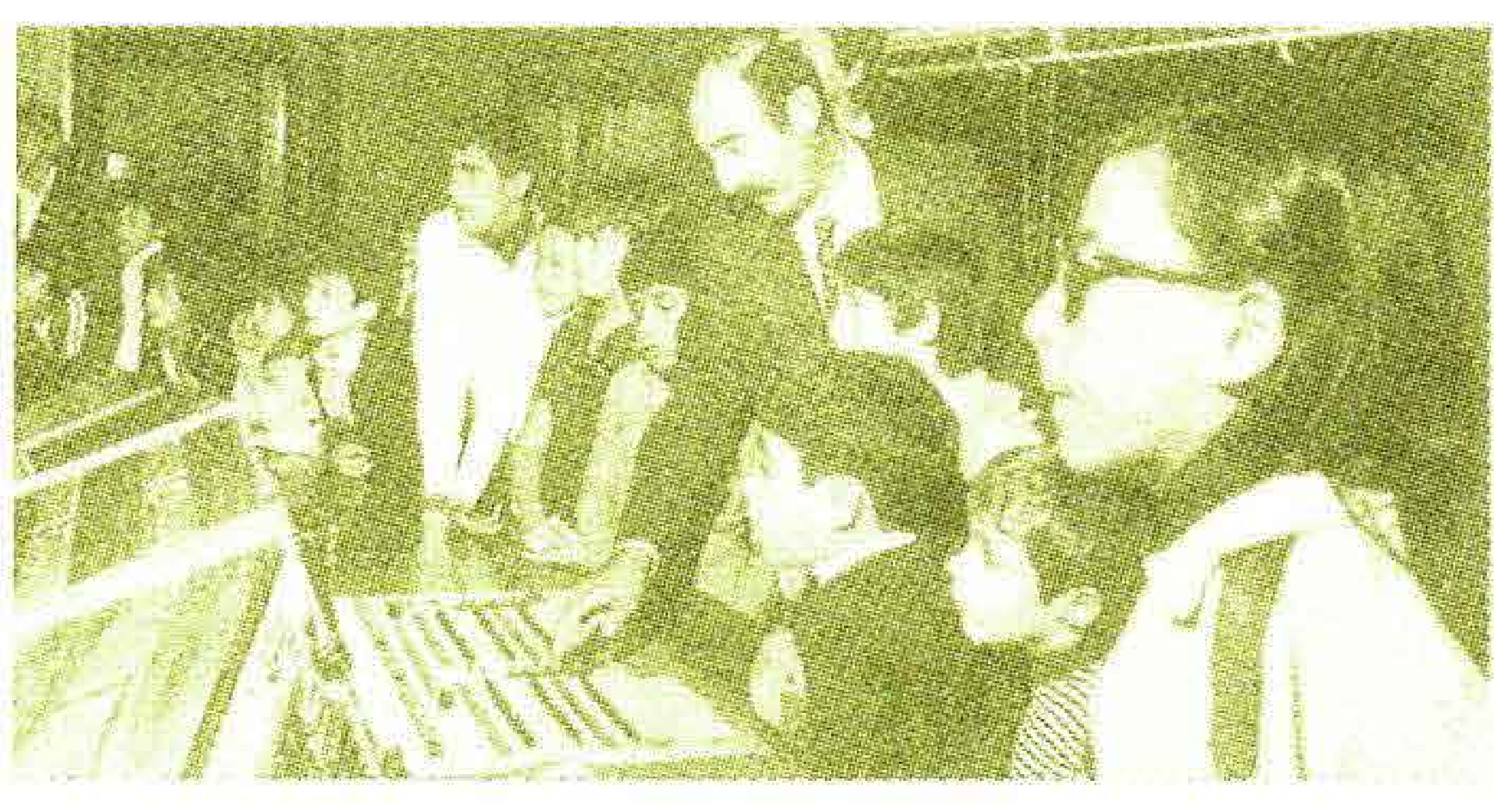

\section{Puntos familia}

Lugares de encuentro para niños de cero a seis años y para los acompañantes adultos -padres, abuelos, hermanos, baby-sitters-. Este serviçio es propuesto sobre todo a las personas que no utilizan los servicios de infancia y ofrece momentos y áreas para que adultos y niños puedan encontrarse, jugar y conocerse intercambiando experiencias de vida y de crecimiento.

\section{Actividades}

- Los adultos y los niños están llamados a participar activamente en la programación y realización de las experiencias educativas.

- Discusión y análisis de asuntos relacionados con las distintas maneras de ser, en lo que se refiere a los niños.

\section{Centros de masaje infantil}

Actividad para público externo, padres con niños de 0 a 3 años, niños minusválidos, -hasta 6 años-, para enseñar a utilizar el masaje -aplicando el método AIMI-, con el propósito de mejorar la relación padreniño utilizando la comunicación no verbal.

\section{Seminario ciudadanos}

En una sociedad que mezcla cada aspecto y experiencia de la vida moderna, también la familia re- gistra cambios profundos. A través de seminarios temáticos sobre educación, se llega a la propuesta de una cultura nueva que preste atención a la familia y su descubrimiento como recurso social.

La página Web termina con el "Laboratorio della Città sostenibile delle bambine e dei bambini". En el sitio se lee:

"La ciudad ideal es aquella en la que un niño no tiene miedo cuando se pierde: pensar en una ciudad más amiga de los niños representa una manera eficaz de imaginarla ideal para todos. El proyecto "Ciudad Sostenible de las Niñas y de los Niños" coloca y define su propia identidad en el ámbito de estas coordenadas. Es un instrumento para realizar acciones dirigidas a reconocer el derecho de los niños a vivir en el ámbito de su comunidad en un clima de relaciones importantes y en una dimensión sostenible; sin riesgos medioambientales, conectados con la seguridad, los espacios, el tiempo y los ritmos que a menudo hacen de las ciudades unos lugares inadecuados y contrastantes para el crecimiento y un desarrollo respetuoso de las exigencias y necesidades de sus habitantes".

Desde 1999 el Laboratorio se ocupa de recoger material y de participar en el Premio Ciudades Sostenibles de las Niñas y de los Niños, instituido por el 


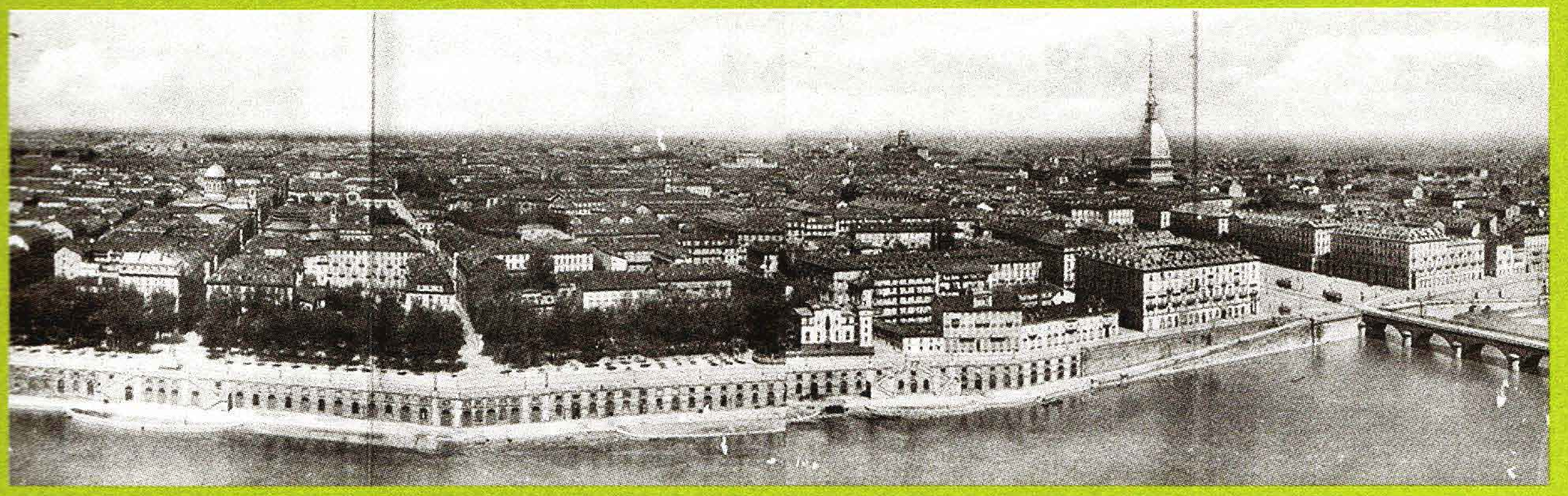

Ministerio del Medio Ambiente y de la Tutela del Territorio. En la primera edición del Premio obtuvo el segundo lugar en el ranking nacional y alcanzó el primer premio en 2001; el Premio fue entregado a la Ciudad de San Giuliano di Puglia, a favor de la construcción de obras para la infancia luego del terremoto que se produjo allí.

La dimensión operativa actual del Laboratorio preyé una actividad de promoción y enlace entre escuelas, asociaciones y sectores de la administración pública para crear itinerarios de análisis del territorio urbano, y del proyecto en que se participe, en lo que se refiere a las dimensiones que más interaccionan con la vida cotidiana de las niñas y los niños: los patios de las escuelas, los espacios frente de las escuelas, los recorridos itinerarios casa-escuela-, y las áreas de juego.

A este propósito, desde el año escolar 2003. 2004 el Proyecto está en su fase operativa, desarro- llando con niños y adolescentes de diecinueve escuelas, programas de "proyecto participado", de los espacios que ellos viven y utilizan, a partir del mismo edificio escolar hasta llegar a toda la aglomeración urbana. La actividad de realización de proyectos reunirá a los técnicos municipales que se ocupan de construcción escollar, viabilidad, verde y medioambiente, y a los niños en su papel de actores directos de los procesos y de las fases proyectivas. Para concretar la actividad descrita de la mejor manera, los alumnos de las escuelas recibirán ayuda y asistencia de un sujeto nuevo, nacido en el seno del Memorándum de Acuerdo entre la Ciudad de Turín y el Colegio de Arquitectos: el Arquitecto de los niños, que, por un lado, tendrá la función de tutor en la actividad proyectiva realizada por los niños y, por otro, jugará el papel de elemento de conexión operativa con los técnicos y las oficinas municipales. 
En lo que se refiere al año escolar 2004-2005, se prevén acciones que se llevarán a cabo en treinta y dos escuelas italianas.

"Crescere in città" es el proyecto dedicado directamente a la relación entre escuela y ciudad, entre los niños-adolescentes y la ciudad. Muchos otros elementos de las políticas municipalęs se añaden y se enfrentan con el proyecto, para hacer de Turín una ciudad educativa. Como antecedente existe el "Proyecto juventud”, que desde 1977 ha hecho de Turín la ciudad piloto de Italia en cuanto a políticas juveniles. Se trata de un proyecto que, con los servicios informativos del centro y de las afueras, considera acciones relacionadas con el trabajo, los intercambios internacionales, la producción cultural, el turismo juvenil y actividades en las cárceles. Existe también el proyecto "Tempi e orari della città", con el que se pretende coordinar la revisión de los tiempos de los servicios públicos, de las escuelas y de las actividades comerciales con el objeto de satisfacer de la manera más completa posible las exigencias de los ciudadanos en general y de las mujeres en particular.
El portal www.torinocultura.it, del cual he sido responsable en estos últimos años, día tras día entrega informaciones útiles para que todos los ciudadanos puedan beneficiarse de la gran oferta cultural que caracteriza nuestra ciudad y de las muchas facilidades previstas para los ciudadanos, como la "Carta musei", que permite la entrada a 140 museos de la región y a las exposiciones organizadas en su interior, a entradas gratis para los jóvenes, mayores, familias y minusválidos. Gran cantidad de otros proyectos y entidades contribuyen para convertir a Turín en una Ciudad Educadora, como la Società Metropolitana delle Acque di Torino, SMAT; el Gruppo Torino Trasporti, GTT; la Azienda Energetica Municipale, AEM; la Azienda Multiservizi Igiene Ambientale Torino, AMIAT, y muchas empresas privadas.

Por todo esto, quiero destacar que en Turín, la experiencia de la Ciudad Educadora se ha adelantado respecto al ámbito de la escuela y de la pedagogía, convirtiéndose en una verdadera perspectiva política.

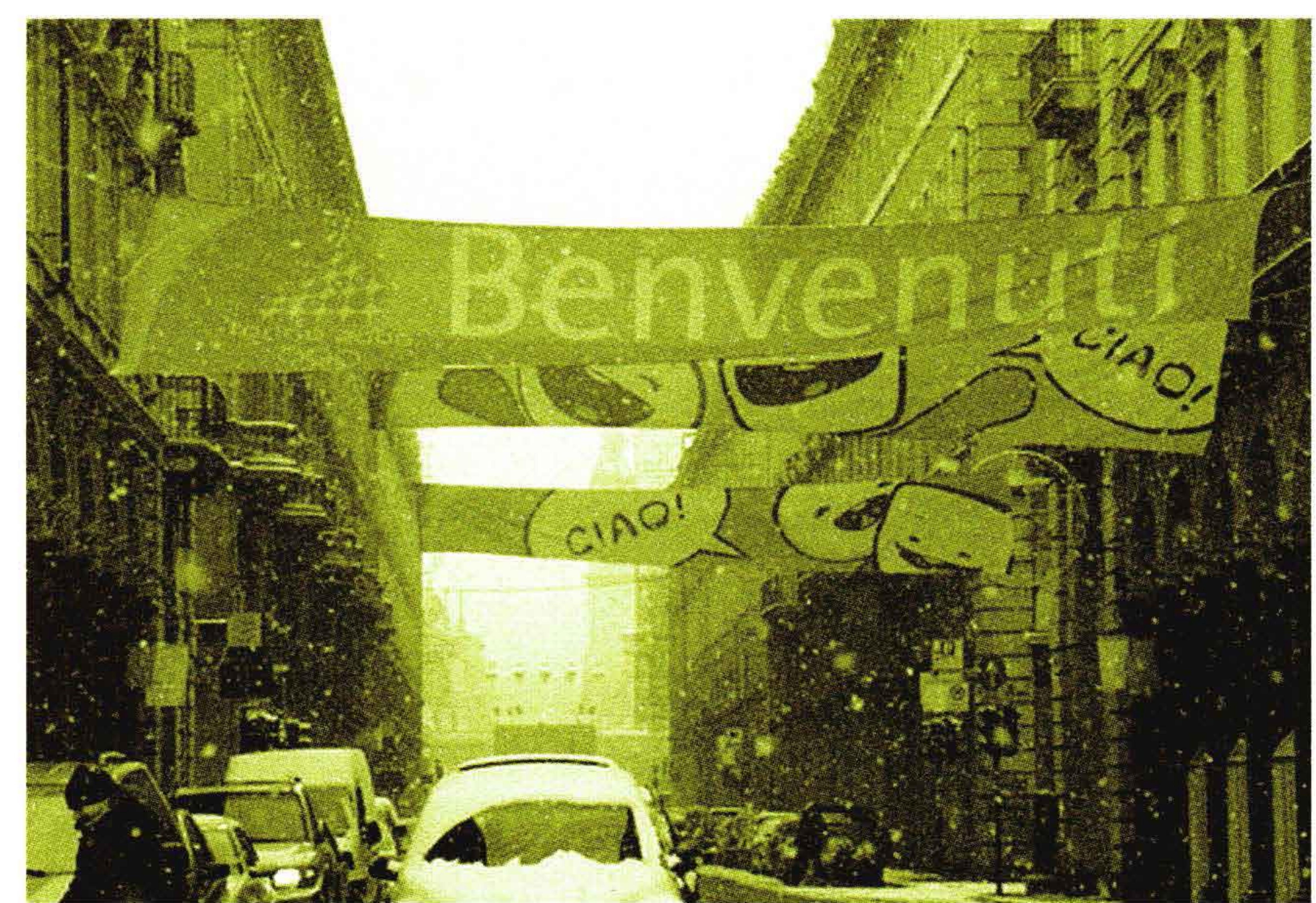

